\title{
Visualização de dados do Índice de Qualidade da Água aplicado a múltiplos pontos em um Sistema de Informação Ambiental
}

\author{
Vania Elisabete Schneider ${ }^{1}$, Odacir Deonisio Graciolli ${ }^{2}$, Helena Graziottin \\ Ribeiro $^{2}$, Adriano Gomes da Silva ${ }^{1}$, Mayara Cechinatto ${ }^{1}$
}

${ }^{1}$ Instituto de Saneamento Ambiental - Universidade De Caxias do Sul (UCS)

Rua Francisco Getúlio Vargas, 1130 - 95070-560 - Caxias do Sul - RS - Brasil

${ }^{2}$ Universidade De Caxias do Sul (UCS)

Rua Francisco Getúlio Vargas, 1130 - 95070-560 - Caxias do Sul - RS - Brasil

\{veschnei, odgracio,hgrib, agsilval1, mcechinatto\} @ucs.br

\begin{abstract}
Information Systems may be configured as tools to support the study and decision making related to environmental issues. Together with databases in a datawarehouse format, these decisions may be geared towards the historical scope of the data. The Environmental Information System - SIA, was developed to store and allow queries of environmental historical data from the Taquari-Antas Hydrographic Basin, in which are installed several hidroeletric plants. This paper presents the development of data visualization capabilities for the Water Quality Index at multiple points in the region.
\end{abstract}

Resumo. Sistemas de Informação podem se configurar como ferramentas para apoio ao estudo e à tomada de decisões relacionadas às questões ambientais. Em conjunto com bancos de dados em formato datawarehouse, estas decisões podem estar voltadas ao âmbito histórico dos dados. O Sistema de Informação Ambiental - SIA, foi desenvolvido para armazenar e permitir a consulta de dados históricos ambientais de diversas centrais hidrelétricas instaladas na Bacia Hidrográfica Taquari-Antas. Esse trabalho apresenta o desenvolvimento de recursos de visualização de dados para o Índice de Qualidade da Água em múltiplos pontos da região.

\section{Introdução}

Informações sobre o meio ambiente tornaram-se mais necessárias e detalhadas na medida em que a sua preservação foi adquirindo importância como política pública ao redor do mundo [GUNTHER 1997]. Dentre os requerentes desse tipo de informação estão as empresas que precisam reportar o seu impacto ambiental aos órgãos de fiscalização. As Tecnologias da Informação (TI) se apresentam como grandes aliadas no armazenamento de dados históricos e no processo de tomada de decisão, uma vez que a informação precisa estar disponível para o gestor em grande escala e de forma condensada [O’BRIAN e MARAKAS 2007].

Para atender as necessidades de armazenamento histórico, consultas considerando diferentes granularidades e exposição aos órgãos de fiscalização as informações ambientais coletadas ao longo de anos por empreendimentos hidrelétricos 
instalados na Bacia Taquari-Antas ${ }^{1}$, localizada a nordeste do estado do Rio Grande do Sul, foi desenvolvido o Sistema de Informações Ambientais - SIA. Os dados utilizados pelo SIA são pertinentes à qualidade da água, ao clima e à fauna da região. Para permitir o armazenamento temporal e processamento analítico, esses dados estão armazenados em um Data Warehouse (DW), um banco de dados que armazena conjuntos de dados históricos de longos períodos para que estes possam ser processados e disponibilizados à gerência com diferentes níveis de detalhe para fornecer indicadores para análise [ELMASRI e NAVATHE 2018], com uma subdivisão em datamarts [KIMBALL e ROSS 2013] separados pelos domínios dos módulos, compartilhando da mesma dimensão tempo. Para tornar mais simples a compreensão dos dados, algo de suma importância para a ciência dos dados e suporte à tomada de decisão [CAO 2017], [MOORE 2017], [BIKAKIS 2018], são utilizados elementos de visualização das informações como relatórios, tabelas, gráficos e um webmapa, para a produção de indicadores, análises estatísticas, consultas a índices e comparações com determinadas legislações ambientais, permitindo a seleção de diferentes filtros de consulta, como agrupamento por regiões e período.

Dentre os índices presentes no sistema está o Índice de Qualidade da Água (IQA), o qual possui o objetivo de avaliar a qualidade da água bruta para sua disponibilização para o abastecimento público após o tratamento [VON SPERLING 2007]. Seus parâmetros são, em sua maioria, indicadores de contaminação causada pelo lançamento de esgotos domésticos [ANA 2018].

Utilizando os dados históricos de monitoramento de qualidade da água armazenados no DW do SIA, este trabalho tem por objetivo apresentar o desenvolvimento de recursos para a visualização agrupada do IQA de diferentes pontos de monitoramento presentes no módulo de qualidade da água. A necessidade de se desenvolver uma ferramenta através da qual o IQA possa ser estudado de forma agrupada e em diferentes pontos da bacia se deve à importância da visualização de dados de indicadores em modo comparativo para a tomada de decisões.

\section{Metodologia}

O SIA é uma aplicação acessível pela web e utiliza a estrutura cliente-servidor [SOMMERVILLE 2011]. No lado servidor o desenvolvimento foi na linguagem PHP. No lado cliente são utilizadas as linguagens HTML, CSS e de programação Javascript para processar as requisições e enviar dados ao lado servidor. Algumas bibliotecas do Javascript são utilizadas para visualização de dados, como C3.js para gráficos gerados dinamicamente e JQGrid para a tabela com dados provenientes das consultas sobre 0 IQA dos pontos.

O armazenamento de dados do sistema é no lado servidor, com a utilização do SGBD PostgreSQL. A estrutura do banco segue o padrão de um DW floco de neve [KIMBALL 2013], com vistas a eventualmente permitir a consulta utilizando diferentes granularidades sobre a dimensão de tempo. Seus domínios estão subdivididos em datamarts para água, clima e fauna, com diversas tabelas fato e dimensão para cada domínio. Para este trabalho a tabela fato utilizada foi a de medições de qualidade da água, com registros datando do ano 2000 até 2019. Nela estão dispostas colunas de informação temporal (data da coleta, data de inserção e data de análise), campos pertinentes aos valores, limites destes valores e campos relacionados a tabelas dimensão

1 Agradecemos as empresas Brookfield, Ceran, Certel e Hidrotérmica pelo fomento ao contínuo desenvolvimento do SIA e pelo apoio à pesquisa. 
pertinentes ao ponto de coleta, qual parâmetro foi coletado, qual o método de análise e qual o de coleta, além do laboratório responsável.

O processamento destes dados e o cálculo do IQA ocorre no lado servidor a cada consulta, em vistas do número de pontos de monitoramento de água presentes no sistema e na existência de campanhas de monitoramento contínuas, sendo esta uma funcionalidade implementada após a estruturação do SIA e do DW. Após isto, os resultados são fornecidos ao gráfico gerado com a biblioteca C3.js e ocorre seu envio ao lado cliente, junto da tabela de consulta, utilizando os mesmos dados que serão dispostos no gráfico.

\section{Resultados}

A ferramenta desenvolvida, intitulada IQA Multipontos, é constituída inicialmente de uma tabela de seleção de pontos (Figura 1), alimentada com dados dos pontos pertinentes ao módulo de água do SIA por meio de uma consulta ao DW. O usuário pode selecionar os pontos por meio da opção de seleção automática, utilizando os parâmetros especificados em cada uma das duas caixas de seleção, escolhendo um ponto pertencente a um recurso hídrico, município, sub-bacia ou empreendimento de sua escolha, com vistas a permitir diferentes granularidades de consulta. Esta seleção passa por um processamento em Javascript, no lado cliente, e então é feita uma requisição ao lado servidor, onde uma classe controladora em PHP solicita ao DW os dados necessários e retorna-os ao lado cliente, onde alimentam uma tabela. Neste caso, ocorre na consulta a verificação no banco se as coordenadas de localização do ponto estão contidas nos pontos geográficos que formam o polígono do ponto escolhido no DW. Além disso, o usuário pode selecionar manualmente os pontos desejados.

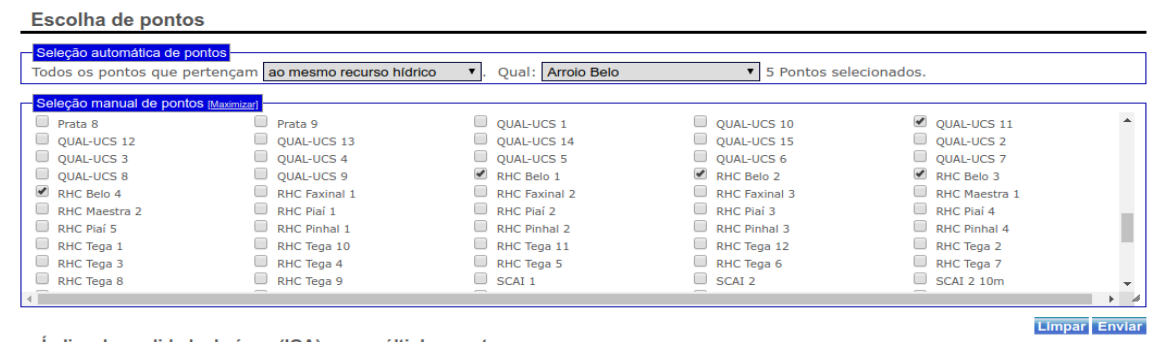

Figura 1. Tabela de seleção de pontos.

Após o envio da consulta, são gerados os componentes de visualização dos resultados (Figura 2). Para esta geração ocorre a aplicação do cálculo do IQA sobre os pontos solicitados, com dados provenientes do DW. O gráfico apresenta historicamente 0 resultado deste cálculo para os diferentes pontos de amostragem selecionados, ou seja, para cada ponto de amostragem é gerado uma linha no gráfico, onde cada ponto sobre ela é uma coleta com um valor de IQA calculado, em função do tempo, disposto no eixo $\mathrm{X}$. Ao posicionar o mouse sobre um determinado ponto no gráfico, é possível visualizar essas informações para a coleta selecionada e para as outras realizadas na mesma data. Vale destacar que os pontos de amostragem variam quanto a quantidade e periodicidade das coletas. As diferentes faixas de cores presentes atrás do gráfico enquadram cada IQA calculado em uma classificação dos recursos hídricos de acordo com a Resolução CONAMA 357, de acordo com os valores dos parâmetros de qualidade da água. A tabela gerada, apresentada na Figura 2, é uma outra forma de exibição dos dados históricos presentes no gráfico. Nela o usuário pode visualizar o IQA calculado para cada campanha de monitoramento presente no gráfico, com o mesmo padrão de cores aplicado sobre o gráfico. 


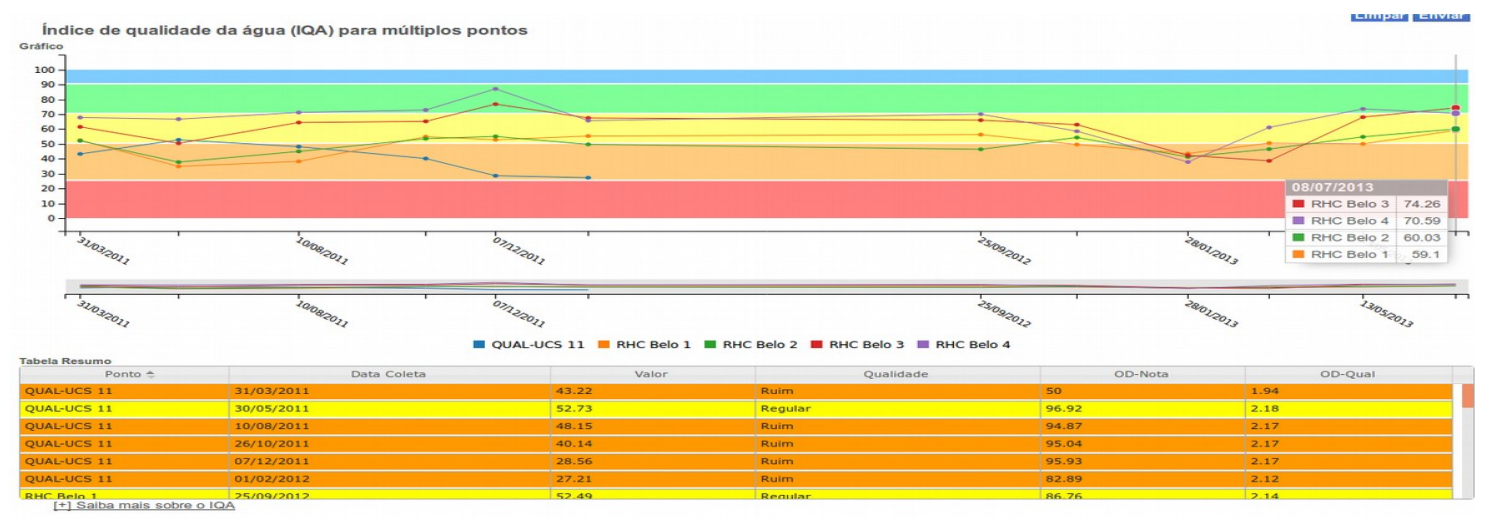

Figura 2. Componentes de visualização dos resultados.

\section{Considerações Finais}

A funcionalidade desenvolvida permite ao usuário a visualização agrupada do IQA em múltiplos pontos. Desta forma, foi facilitada a comparação da qualidade da água em diferentes pontos da Bacia Hidrográfica. Essa vantagem diminui o tempo de trabalho do gestor ou pesquisador que busca utilizar dessas informações em suas pesquisas e estudos relacionados ao meio ambiente da região, aumentando a eficiência do SIA como ferramenta de apoio a gestão ambiental e a geração de conhecimento. Futuramente, pretende-se inserir à funcionalidade um filtro de consulta temporal, no qual o usuário poderá definir a data de início e fim do período para o qual deseja gerar o gráfico. Além disso, pretende-se acrescentar a visualização em múltiplos pontos para outros índices presentes no SIA.

\section{Referências}

ANA - Agência Nacional de Águas. Indicadores de Qualidade - Índice de Qualidade das Águas (IQA). Disponível em: <http://pnqa.ana.gov.br/indicadores-indice-aguas.asp x>. Acesso em: 08 ago. 2018.

Bikakis, N. Big Data Visualization Tools Encyclopedia of Big Data Technologies, Springer 2018. Disponível em: <https://arxiv.org/pdf/1801.08336.pdf>. Acesso em: 18 fev. 2019.

Günther, O. Environmental information systems. Acm Sigmod Record, v. 26, n. 1, p.3-4, mar. 1997.

Kimball, R. e Ross, M. The Data Warehouse Toolkit: The Definitive Guide to Dimensional Modeling. 3. ed. Editora: John Wiley \& Sons, 2013. 564 p.

Von Sperling, M. Estudos e modelagem da qualidade da água de rios. 1 ed. v. 7. Belo Horizonte: Departamento de Engenharia Sanitária e Ambiental; Universidade Federal de Minas Gerais, 2007

O’Brien, J. A. e Marakas, G. M. Management Information Systems. Dias Technology Review, v. 4, n. 2, p.102-112, out. 2008.

Sommerville, I. Engenharia de Software. 9. ed. São Paulo: Pearson Prentice Hall, 2011. Elmasri, R. e Navathe, S.B. Sistemas de Bancos de Dados. 7. ed. São Paulo: Pearson Education do Brasil, 2018. 1127 p.

Cao, L. Data science. Communications Of The Acm, [s.l.], v. 60, n. 8, p.59-68, 24 jul. 2017. Association for Computing Machinery (ACM). http://dx.doi.org/10.1145/3015456.

Moore, J. Data Visualization in Support of Executive Decision Making. Interdisciplinary Journal Of Information, Knowledge, And Management, [s.l.], v. 12, p.125-138, 2017. Informing Science Institute. http://dx.doi.org/10.28945/3687. 